\title{
Event-Triggered Mean-Square Consensus Control for Time-Varying Stochastic Multi-Agent System with Sensor Saturations
}

\author{
Lifeng Ma, Zidong Wang, Fellow, IEEE, and Hak-Keung Lam
}

\begin{abstract}
In this paper, the consensus control problem is investigated for a class of discrete time-varying stochastic multiagent system subject to sensor saturations. An event-based mechanism is adopted where each agent updates the control input signal only when the pre-specified triggering condition is violated. To reflect the time-varying manner and characterize the transient consensus behavior, a new index for mean-square consensus is put forward to quantify the deviation level from individual agent to the average value of all agents' states. For a fixed network topology, the aim of the proposed problem is to design time-varying output-feedback controllers such that, at each time step, the mean-square consensus index of the closedloop multi-agent system satisfies the pre-specified upper bound constraints subject to certain triggering mechanism. Both the existence conditions and the explicit expression of the desired controllers are established by resorting to the solutions to a set of recursive matrix inequalities. An illustrative simulation example is utilized to demonstrate the usefulness of the proposed algorithms.
\end{abstract}

Index Terms-Multi-agent systems; Time-varying systems; Mean-square consensus; Event-triggered control; Sensor saturations.

\section{INTRODUCTION}

The past decade has witnessed an ever-growing interest in the study of so-called multi-agent systems (MASs) that have found extensive applications in various areas including unmanned aerial vehicles (UAVs) [22], autonomous underwater vehicles (AUVs) [21], automated highway systems (AHSs) [2] and mobile robotics [23]. Among popular research issues regarding MASs, the so-called consensus problem is concerned with the process where a batch of interacting agents governed by certain interconnection topology achieve a collective goal (e.g. the same trajectory). Owing to their clear engineering insights, the consensus behaviors of MASs have attracted a

This work was supported in part by the Royal Society of the U.K., the National Natural Science Foundation of China under Grants 61304010 and 61329301, the Natural Science Foundation of Jiangsu Province under Grant BK20130766, the Postdoctoral Science Foundation of China under Grant 2014M551598, International Postdoctoral Exchange Fellowship from the China Postdoctoral Council, the Fundamental Research Funds for the Central Universities under Grant 30916011205, and Alexander von Humboldt Foundation of Germany.

L. Ma is with the School of Automation, Nanjing University of Science and Technology, Nanjing 210094, China. (Email: malifeng@njust . edu. cn)

Z. Wang is with the Department of Computer Science, Brunel University London, Uxbridge, Middlesex, UB8 3PH, United Kingdom. (Email: Zidong.Wang@brunel.ac.uk)

H.-K. Lam is with Department of Informatics, School of Natural \& Mathematical Science, King's College London, Strand Campus, WC2R 2LS, United Kingdom. surge of research attention leading to a rich body of literature, see [4], [9], [10], [18], [19] and the references therein.

Up to now, most MASs discussed in the literature have been assumed to be time-invariant. This assumption is, however, very restrictive as almost all real-world engineering systems have certain parameters/structures which are indeed timevarying [1]. For such time-varying systems, a finite-horizon controller is usually desirable as it could provide better transient performance for the controlled system especially when the noise inputs are non-stationary, see [6], [8] for some recent results. However, when it comes to the consensus of multiagent systems, the corresponding results have been scattered due mainly to the difficulty in quantifying the consensus over a finite horizon. It is notable that the consensus problem for MASs with time-varying parameters has received some initial research attention (see e.g. [11], [14], [26]). Nevertheless, the research on time-varying multi-agent systems is far from adequate and there are still many open challenging problems remaining for further investigation. On the other hand, the sensor saturation is a frequently encountered phenomenon resulting from physical limitations of system components as well as the difficulties in ensuring high fidelity and timely arrival of the control and sensing signals through a possibly unreliable network of limited bandwidth. In other words, the sensor outputs are often saturated because the physical entities or processes cannot transmit energy and power with unbounded magnitude or rate. As such, it makes practical sense to take the sensor saturation into account when dealing with the output-feedback control problems for time-varying MASs, which remains as an ongoing research issue.

On another research frontier, the event-triggered control/filtering strategies have recently become an attractive area of research because of their capabilities in improving the resource utilization efficiency by reducing the unnecessary executions as compared to the traditional time-triggered mechanism, see [5], [6], [9], [10], [13], [17] and the references therein. In the context of MASs, so far, much work has been done for event-triggered consensus control and most available results have been restricted to linear time-invariant MASs only, see [7], [20] for instances. When it comes to the timevarying MASs, the corresponding event-triggered schemes have received very little research effort due probably to the technical difficulty in handling the time-varying coupling between the triggering mechanism and interaction topology in case of communications among the agents. To the best of our knowledge, the mean-square consensus control problem for 
time-varying MASs with event-triggered mechanism has not been adequately investigated, not to mention the case where the sensor saturations are also involved. Such a situation has motivated the present investigation.

In this paper, we endeavor to design an event-triggered output-feedback controller for a class of discrete time-varying stochastic MAS to reach a new kind of mean-square consensus with guaranteed upper bound on the consensus index subject to sensor saturations. In doing so, three technical challenges are identified as follows: 1) how to define the consensus of MASs in a time-varying context; 2) how to develop appropriate analysis and synthesis techniques associated with time-varying MASs; and 3) how to establish a unified framework to handle the cross coupling among topology, time-varying parameters, event-triggered mechanism as well as the sensor saturations? In fact, the main purpose of this paper is to provide satisfactory answers to these questions by launching a major study.

The contributions of this paper are outlined as follows: i) the system model is comprehensive that takes stochastic noises, time-varying effect and several engineering-oriented constraints into simultaneous consideration; ii) the newly proposed mean-square consensus index provides an intuitive insight into the characterization of the transient behavior in the consensus process; and iii) the proposed algorithm allows much flexibility in making the trade-off between certain essential performances (i.e., consensus accuracy and triggering frequency).

The rest of this paper is organized as follows. Section II formulates the event-triggered output-feedback consensus control problem for discrete time-varying stochastic MAS subject to sensor saturations. The main results are presented in Section III where sufficient conditions for the MAS to reach the mean-square consensus with guaranteed performance are given in terms of recursive matrix inequalities. Section IV gives a numerical example and Section $\mathrm{V}$ draws our conclusion.

Notation $\mathbb{R}^{n}$ denotes the $n$-dimensional Euclidean space, $\mathbf{1}_{n}$ denotes an $n$-dimensional column vector with all ones, and $I_{n}$ denotes the identity matrix of $n$ dimensions. The notation $X \geq$ $Y$ (respectively $X>Y$ ), where $X$ and $Y$ are symmetric matrices, means that $X-Y$ is positive semi-definite (respectively positive definite). $\mathbb{E}\{x\}$ stands for the expectation of stochastic variable $x$ and $\mathbb{E}\{x \mid y\}$ for the expectation of $x$ conditional on $y$. For matrices $A \in \mathbb{R}^{m \times n}$ and $B \in \mathbb{R}^{p \times q}$, their Kronecker product is a matrix in $\mathbb{R}^{m p \times n q}$ denoted as $A \otimes B$. $\operatorname{tr}[A]$ means the trace of matrix $A$ and $\operatorname{diag}\left\{F_{1}, F_{2}, \ldots, F_{n}\right\}$ denotes a block diagonal matrix whose diagonal blocks are given by $F_{1}, F_{2}, \ldots, F_{n}$. The notation $\operatorname{col}_{n}\left\{x_{i}\right\}$ represents the column vector $\left[\begin{array}{llll}x_{1}^{\mathrm{T}} & x_{2}^{\mathrm{T}} & \cdots & x_{n}^{\mathrm{T}}\end{array}\right]^{\mathrm{T}} \cdot \operatorname{diag}_{n}\{A\}$ and $\operatorname{diag}_{n}\left\{A_{i}\right\}$ denote the block diagonal matrices $\operatorname{diag}\{A, A, \ldots, A\}$ and $\operatorname{diag}\left\{A_{1}, A_{2}, \ldots, A_{n}\right\}$, respectively.

\section{Problem Formulation}

In this paper, the multi-agent system has $N$ agents which communicate with each other according to a fixed network topology represented by an undirected graph $\mathscr{G}=(\mathscr{V}, \mathscr{E}, \mathscr{H})$ of order $N$ with the set of agents $\mathscr{V}=\{1,2, \ldots, N\}$, the set of edges $\mathscr{E} \in \mathscr{V} \times \mathscr{V}$, and the weighted adjacency matrix $\mathscr{H}=$ $\left[h_{i j}\right]$ with nonnegative adjacency element $h_{i j}$. If $(i, j) \in \mathscr{E}$, then $h_{i j}>0$, else $h_{i j}=0$. An edge of $\mathscr{G}$ is denoted by the ordered pair $(i, j)$. The adjacency elements associated with the edges of the graph are positive, i.e., $h_{i j}>0 \Longleftrightarrow(i, j) \in \mathscr{E}$, which means that agent $i$ can obtain information from agent $j$. Furthermore, self-edges $(i, i)$ are not allowed, i.e., $(i, i) \notin \mathscr{E}$ for any $i \in \mathscr{V}$. The neighborhood of agent $i$ is denoted by $\mathscr{N}_{i}=\{j \in \mathscr{V}:(j, i) \in \mathscr{E}\}$. The in-degree of agent $i$ is defined as $\operatorname{deg}_{\text {in }}^{i} \triangleq \sum_{j \in \mathscr{N}_{i}} h_{i j}$.

Consider a discrete time-varying stochastic multi-agent system described by the following state-space model:

$$
\begin{aligned}
x_{i, k+1} & =A_{k} x_{i, k}+B_{k} u_{i, k}+D_{k} w_{i, k}, \\
y_{i, k} & =\kappa\left(C_{k} x_{i, k}\right)+E_{k} v_{i, k},
\end{aligned}
$$

where $x_{i, k} \in \mathbb{R}^{n}, y_{i, k} \in \mathbb{R}^{q}$ and $u_{i, k} \in \mathbb{R}^{p}$ are, respectively, the state vector, the measurement output and the control input of agent $i . A_{k}, B_{k}, C_{k}, D_{k}$ and $E_{k}$ are timevarying matrices with compatible dimensions. $w_{i, k} \in \mathbb{R}^{\omega}$ and $v_{i, k} \in \mathbb{R}^{\nu}(i=1,2, \ldots, N)$ are mutually uncorrelated zero-mean Gaussian white noise sequences. Denote $\tilde{w}_{k} \triangleq$

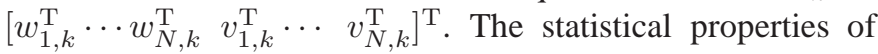
$w_{i, k}$ and $v_{i, k}$ can be described as follows:

$$
\begin{aligned}
\mathbb{E}\left\{\tilde{w}_{k}\right\} & =0, \\
\mathbb{E}\left\{\tilde{w}_{k} \tilde{w}_{l}^{\mathrm{T}}\right\} & =\left[\begin{array}{cc}
\operatorname{diag}_{N}\left\{W_{i, k} \delta_{k l}\right\} & 0 \\
0 & \operatorname{diag}_{N}\left\{V_{i, k} \delta_{k l}\right\}
\end{array}\right]
\end{aligned}
$$

where $W_{i, k}$ and $V_{i, k}(i=1,2, \ldots, N)$ are all known positive definite matrices, and $\delta_{k l}$ is defined by:

$$
\delta_{k l}= \begin{cases}I & k=l \\ 0 & k \neq l\end{cases}
$$

The saturation function $\kappa(\cdot)$ in (2) is defined as

$$
\kappa(r) \triangleq \operatorname{col}_{q}\left\{\kappa_{i}\left(r^{(i)}\right)\right\}
$$

where $\kappa_{i}\left(r^{(i)}\right)=\operatorname{sign}\left(r^{(i)}\right) \min \left\{r_{\max }^{(i)},\left|r^{(i)}\right|\right\}$ with $r^{(i)}$ denoting the $i$ th entry of the vector $r$.

Definition 1: Let $U_{1}$ and $U_{2}$ be real matrices with $U \triangleq$ $U_{2}-U_{1}>0$. A nonlinearity $\varphi(\cdot)$ is said to satisfy the sector condition with respect to $U_{1}$ and $U_{2}$ if

$$
\left(\varphi(y)-U_{1} y\right)^{\mathrm{T}}\left(\varphi(y)-U_{2} y\right) \leq 0 .
$$

In this case, the sector-bounded nonlinearity $\varphi(\cdot)$ is said to belong to the sector $\left[U_{1}, U_{2}\right]$.

Noting that if there exist diagonal matrices $G_{1}$ and $G_{2}$ such that $0 \leq G_{1}<I \leq G_{2}$, then the saturation function $\kappa\left(C_{k} x_{i, k}\right)$ in (2) can be written as follows:

$$
\kappa\left(C_{k} x_{i, k}\right)=G_{1} C_{k} x_{i, k}+\varphi\left(C_{k} x_{i, k}\right)
$$

where $\varphi\left(C_{k} x_{i, k}\right)$ is a nonlinear vector-valued function satisfying the sector condition with $U_{1}=0$ and $U_{2}=G \triangleq G_{2}-G_{1}$, i.e., $\varphi\left(C_{k} x_{i, k}\right)$ satisfies the following inequality:

$$
\varphi^{\mathrm{T}}\left(C_{k} x_{i, k}\right)\left(\varphi\left(C_{k} x_{i, k}\right)-G C_{k} x_{i, k}\right) \leq 0 .
$$


In this paper, a control protocol of the following form is adopted:

$$
u_{i, k}=K_{k} \eta_{i, k} \quad \text { with } \quad \eta_{i, k}=\sum_{j \in \mathscr{N}_{i}} h_{i j}\left(y_{j, k}-y_{i, k}\right)
$$

where $K_{k}$ is the feedback gain to be designed and $\eta_{i, k}$ represents the updating signal feeding to the controller of agent $i$.

Let us now discuss the event-triggering mechanism to be adopted. Suppose that the sequence of the triggering instants is $\left\{k_{t}^{i}\right\}(t=0,1,2, \ldots)$ satisfying $0<k_{0}^{i}<k_{1}^{i}<k_{2}^{i}<\cdots<$ $k_{t}^{i}<\cdots$, where $k_{t}^{i}$ represents the time instant $k$ when the $(t+1)$-th trigger occurs for agent $i$. Then, for $k>k_{t}^{i}$, define

$$
e_{i, k} \triangleq \eta_{i, k_{t}^{i}}-\eta_{i, k}
$$

with $\eta_{i, k_{t}^{i}}$ representing the updating signal feeding to the controller of agent $i$ at the latest triggering time $k_{t}^{i}$. Then, the sequence of event-triggering instants is determined iteratively by

$$
k_{t+1}^{i}=\inf \left\{k \in \mathbb{Z}^{+} \mid k>k_{t}^{i}, \quad e_{i, k}^{\mathrm{T}} \Omega_{i, k}^{-1} e_{i, k}>1\right\}
$$

where $\Omega_{i, k}>0$ is referred to as the triggering threshold matrix.

Applying the event-triggering mechanism, we can rewrite the updating signal $\eta_{i, k}$ defined in (7) as follows:

$$
\eta_{i, k}= \begin{cases}0, & k \in\left[0, k_{0}^{i}\right) \\ \eta_{i, k_{t}^{i}}, & k \in\left[k_{t}^{i}, k_{t+1}^{i}\right)\end{cases}
$$

which implies that the controller input defined in (7) remains a constant in the execution interval $\left[k_{t}, k_{t+1}\right)$. Without loss of generality, it is assumed that $\eta_{i, k_{t}^{i}}=0$ when $k \in\left[\begin{array}{ll}0 & k_{0}^{i}\end{array}\right)$. The control law can be now rewritten as

$$
u_{i, k}=K_{k}\left(\eta_{i, k}+e_{i, k}\right), \quad e_{i, k}^{\mathrm{T}} \Omega_{i, k}^{-1} e_{i, k} \leq 1 .
$$

Implementing control law (11) to MAS (1)-(2), we obtain the following closed-loop system:

$$
\begin{aligned}
x_{k+1}= & \left(I_{N} \otimes A_{k}+\mathcal{H}_{k} \otimes\left(B_{k} K_{k} G_{1} C_{k}\right)\right) x_{k} \\
& +\left(I_{N} \otimes D_{k}\right) w_{k}+\left(\mathcal{H}_{k} \otimes\left(B_{k} K_{k} E_{k}\right)\right) v_{k} \\
& +\left(\mathcal{H}_{k} \otimes\left(B_{k} K_{k}\right)\right) \varphi_{k}+\left(I_{N} \otimes\left(B_{k} K_{k}\right)\right) e_{k}
\end{aligned}
$$

where

$$
\begin{aligned}
x_{k} & =\operatorname{col}_{N}\left\{x_{i, k}\right\}, e_{k}=\operatorname{col}_{N}\left\{e_{i, k}\right\}, \varphi_{k}=\operatorname{col}_{N}\left\{\kappa\left(C_{k} x_{i, k}\right)\right\}, \\
w_{k} & =\operatorname{col}_{N}\left\{w_{i, k}\right\}, v_{k}=\operatorname{col}_{N}\left\{v_{i, k}\right\} \\
\mathcal{H}_{k} & =\left[\begin{array}{ccccc}
-\operatorname{deg}_{\text {in }}^{1} & h_{1,2} & h_{1,3} & \cdots & h_{1, N} \\
h_{2,1} & -\operatorname{deg}_{\text {in }}^{2} & h_{2,3} & \cdots & h_{2, N} \\
\vdots & \vdots & \vdots & \ddots & \vdots \\
h_{N, 1} & h_{N, 2} & h_{N, 3} & \cdots & -\operatorname{deg}_{\text {in }}^{N}
\end{array}\right]
\end{aligned}
$$

In order to discuss the consensus performance of MAS (1)(2) in the mean square, we first denote the average state of all agents by:

$$
\bar{x}_{k} \triangleq \mathbb{E}\left\{\frac{1}{N} \sum_{i=1}^{N} x_{i, k} \mid y_{k-1}\right\}=\frac{1}{N} \mathbb{E}\left\{\left(\mathbf{1}_{N}^{\mathrm{T}} \otimes I_{n}\right) x_{k} \mid y_{k-1}\right\}
$$

where $y_{k-1} \triangleq \operatorname{col}_{N}\left\{y_{i, k-1}\right\}$ represents the measurements obtained at time instant $k-1$. Then, at time instant $k$, given the measurements $y_{k} \triangleq \operatorname{col}_{N}\left\{y_{i, k}\right\}$ (which means that $e_{k}$ is also available according to (7)-(8)), we can calculate $\bar{x}_{k+1}$ by

$$
\begin{aligned}
\bar{x}_{k+1} & =\frac{1}{N} \mathbb{E}\left\{\left(\mathbf{1}_{N}^{\mathrm{T}} \otimes I_{n}\right) x_{k+1} \mid y_{k}\right\} \\
& =A_{k} \bar{x}_{k}+\frac{1}{N}\left(\mathbf{1}_{N}^{\mathrm{T}} \otimes\left(B_{k} K_{k}\right)\right) e_{k} .
\end{aligned}
$$

It should be pointed out that recursion (14) plays a pivotal role in computing the expected average state at each time instant and subsequently in obtaining the desired feedback gain. Such a procedure will be discussed later in more detail.

Definition 2: The performance index of the mean-square consensus for agent $i(i=1,2, \ldots, N)$ of the time-varying stochastic multi-agent system (1)-(2) at time instant $k$ is defined by

$$
\mathfrak{D}_{i, k} \triangleq \mathbb{E}\left\{\left(x_{i, k}-\bar{x}_{k}\right)\left(x_{i, k}-\bar{x}_{k}\right)^{\mathrm{T}}\right\} .
$$

Remark 1: The performance index $\mathfrak{D}_{i, k}$ of the mean-square consensus characterizes the deviation level from the agent $i$ to the expected average of the states $\bar{x}_{k}$ at time instant $k$, thereby reflecting the transient consensus accuracy during the dynamical consensus process. Such an index, which can be intuitively understood as the "distance" from agent $i$ to the expected center of the MAS (characterized by $\bar{x}_{k}$ ) at time step $k$, is proposed in response to the consideration of the additive noises $w_{i, k}$ and $v_{i, k}$, the event-triggered mechanism (9) as well as the time-varying nature of MAS (1)-(2). In general, a smaller $\mathfrak{D}_{i, k}$ (in the sense of matrix trace) is indicative of a better consensus performance at time instant $k$.

Assumption 1: The initial values of each agent, namely, $x_{i, 0}$ $(i=1,2, \ldots, N)$ are known and satisfy

$$
\left(x_{i, 0}-\bar{x}_{0}\right)\left(x_{i, 0}-\bar{x}_{0}\right)^{\mathrm{T}} \leq \Gamma_{0}
$$

where $\Gamma_{0}>0$ is a known positive definite matrix.

Definition 3: Let the undirected communication graph $\mathscr{G}$, a sequence of triggering threshold matrices $\left\{\Omega_{i, k}\right\}_{k \geq 0}$ and a sequence of positive definite matrices $\left\{\Gamma_{k}\right\}_{k \geq 0}$ be given. The MAS (1)-(2) is said to reach mean-square consensus with respect to the triple $\left(\mathscr{G},\left\{\Omega_{i, k}\right\},\left\{\Gamma_{k}\right\}\right)$ if

$$
\mathfrak{D}_{i, k} \leq \Gamma_{k}, \quad \forall i \in \mathscr{V}, k \geq 0 .
$$

hold at each time instant $k$, where $\mathfrak{D}_{i, k}$ is defined in (15).

Our objective of this paper is twofold. First, we aim to design the sequence of output-feedback gains $\left\{K_{k}\right\}_{k \geq 0}$ such that MAS (1)-(2) reaches mean-square consensus with respect to $\left(\mathscr{G},\left\{\Omega_{i, k}\right\},\left\{\Gamma_{k}\right\}\right)$. Second, we aim to solve two optimization problems which, respectively, minimize $\Gamma_{k}$ (in the sense of matrix trace) to seek the locally best consensus performance and maximize $\Omega_{i, k}$ (in the sense of matrix trace) to design the locally lowest triggering frequency at each time instant.

\section{MAIN RESULTS}

Lemma 1: A symmetric matrix $P \in \mathbb{R}^{\epsilon \times \epsilon}$ is positive definite if and only if there exist $\rho_{l} \in \mathbb{R}^{\epsilon}(l=1,2, \ldots, \epsilon)$ such that $P=\sum_{l=1}^{\epsilon} \rho_{l} \rho_{l}^{\mathrm{T}}$ and $\operatorname{rank}\left[\begin{array}{llll}\rho_{1} & \rho_{2} & \cdots & \rho_{\epsilon}\end{array}\right]=\epsilon$. 
Lemma 2: (S-procedure [3]) Let $\psi_{0}(\cdot), \psi_{1}(\cdot), \ldots, \psi_{m}(\cdot)$ be quadratic functions of the variable $\varsigma \in \mathbb{R}^{n}: \psi_{j}(\varsigma) \triangleq \varsigma^{\mathrm{T}} T_{j} \varsigma$ $(j=0, \ldots, m)$, where $T_{j}^{\mathrm{T}}=T_{j}$. If there exist $\tau_{1} \geq 0$, $\ldots, \tau_{m} \geq 0$ such that $\varsigma^{\mathrm{T}}\left(T_{0}-\sum_{j=1}^{m} \tau_{j} T_{j}\right) \varsigma \leq 0$, then the following is true:

$$
\psi_{1}(\varsigma) \leq 0, \ldots, \psi_{m}(\varsigma) \leq 0 \rightarrow \psi_{0}(\varsigma) \leq 0 .
$$

Lemma 3: (Schur Complement Equivalence) Given constant matrices $\mathcal{S}_{1}, \mathcal{S}_{2}, \mathcal{S}_{3}$ where $\mathcal{S}_{1}=\mathcal{S}_{1}^{\mathrm{T}}$ and $0<\mathcal{S}_{2}=\mathcal{S}_{2}^{\mathrm{T}}$, then $\mathcal{S}_{1}+\mathcal{S}_{3}^{\mathrm{T}} \mathcal{S}_{2}^{-1} \mathcal{S}_{3}<0$ if and only if

$$
\left[\begin{array}{cc}
\mathcal{S}_{1} & \mathcal{S}_{3}^{\mathrm{T}} \\
\mathcal{S}_{3} & -\mathcal{S}_{2}
\end{array}\right]<0, \quad \text { or } \quad\left[\begin{array}{cc}
-\mathcal{S}_{2} & \mathcal{S}_{3} \\
\mathcal{S}_{3}^{\mathrm{T}} & \mathcal{S}_{1}
\end{array}\right]<0 .
$$

A. Consensus control subject to a fixed triple $\left(\mathscr{G}, \Omega_{i, k}, \Gamma_{k}\right)$

For simplicity of the following notation, we denote

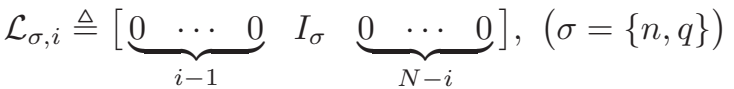

$$
\begin{aligned}
& \mathcal{N}_{k} \triangleq\left[a_{i j}\right]_{N \times N} \text { with } a_{i j} \triangleq \begin{cases}(1-N) / N, & i=j \\
-1 / N, & i \neq j\end{cases} \\
& \mathcal{T}_{1, k} \triangleq \operatorname{diag}\left\{-1, \mathcal{L}_{n, i}^{\mathrm{T}} \mathcal{L}_{n, i}, 0,0\right\}, \mathcal{E}_{k} \triangleq \mathcal{H}_{k} \otimes\left(B_{k} K_{k} E_{k}\right) \text {, } \\
& \mathcal{T}_{2, k} \triangleq \operatorname{diag}\left\{-1,0, \mathcal{L}_{q, i}^{\mathrm{T}} \Omega_{i, k}^{-1} \mathcal{L}_{q, i}, 0\right\}, \mathcal{D}_{k} \triangleq I_{N} \otimes D_{k}, \\
& \mathcal{W}_{k} \triangleq \operatorname{diag}_{N}\left\{W_{i}\right\}, \mathscr{D}_{k} \triangleq \mathcal{D}_{k}^{\mathrm{T}} \mathcal{L}_{n, i}^{\mathrm{T}} \Gamma_{k+1}^{-1} \mathcal{L}_{n, i} \mathcal{D}_{k} \mathcal{W}_{k}, \\
& \mathcal{V}_{k} \triangleq \operatorname{diag}_{N}\left\{V_{i}\right\}, \mathscr{E}_{k} \triangleq \mathcal{E}_{k}^{\mathrm{T}} \mathcal{L}_{n, i}^{\mathrm{T}} \Gamma_{k+1}^{-1} \mathcal{L}_{n, i} \mathcal{E}_{k} \mathcal{V}_{k}, \\
& \bar{\Pi}_{12} \triangleq I_{N} \otimes\left(A_{k} F_{k}\right)+\mathcal{H}_{k} \otimes\left(B_{k} K_{k} G_{1} C_{k} F_{k}\right) \text {, }
\end{aligned}
$$

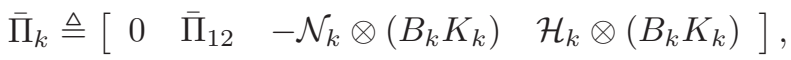

$$
\begin{aligned}
& \bar{\Psi}_{k} \triangleq\left[\begin{array}{lll}
-\left(\mathbf{1}_{N} \otimes\left(G C_{k}\right)\right) \bar{x}_{k} & -I_{N} \otimes\left(G C_{k} F_{k}\right) & 0
\end{array}\right], \\
& \Psi_{k} \triangleq \frac{1}{2}\left[\begin{array}{cc}
0 & \bar{\Psi}_{k}^{\mathrm{T}} \\
\bar{\Psi}_{k} & 2 I_{n N}
\end{array}\right] \text {. }
\end{aligned}
$$

Moreover, by Lemma 1 , the matrices $\mathcal{W}_{k}$ and $\mathcal{V}_{k}$ can be decomposed by $\mathcal{W}_{k}=\sum_{l=1}^{\epsilon} \vartheta_{l, k} \vartheta_{l, k}^{\mathrm{T}}$ and $\mathcal{V}_{k}=\sum_{l=1}^{\varepsilon} \pi_{l, k} \pi_{l, k}^{\mathrm{T}}$ with $\vartheta_{l, k} \in \mathbb{R}^{\epsilon}$ and $\pi_{l, k} \in \mathbb{R}^{\varepsilon}(\epsilon=N \omega, \varepsilon=N \nu)$.

Theorem 1: Let the triple $\left(\mathscr{G},\left\{\Omega_{i, k}\right\},\left\{\Gamma_{k}\right\}\right)$ be given. MAS (1)-(2) reaches mean-square consensus with respect to the triple $\left(\mathscr{G},\left\{\Omega_{i, k}\right\},\left\{\Gamma_{k}\right\}\right)$ if there exist a sequence of real-valued matrices $\left\{K_{k}\right\}_{k \geq 0}$, sequences of positive scalars $\left\{\gamma_{i, k}\right\}_{k \geq 0}$ and $\left\{\lambda_{i, k}\right\}_{k \geq 0}$, sequences of non-negative scalars $\left\{\tau_{i, k}^{(1)}\right\}_{k \geq 0}$, $\left\{\tau_{i, k}^{(2)}\right\}_{k \geq 0}$ and $\left\{\tau_{k}^{(3)}\right\}_{k \geq 0}(i=1,2, \ldots N)$ such that the following recursive linear matrix inequalities (RLMIs) are true:

$$
\begin{gathered}
{\left[\begin{array}{cc}
-\gamma_{i, k} & \bar{\vartheta}_{k}^{\mathrm{T}} \\
\bar{\vartheta}_{k} & -\operatorname{diag}_{\epsilon}\left\{\Gamma_{k+1}\right\}
\end{array}\right] \leq 0,} \\
{\left[\begin{array}{cc}
-\lambda_{i, k} & \bar{\pi}_{k}^{\mathrm{T}} \\
\bar{\pi}_{k} & -\operatorname{diag}_{\varepsilon}\left\{\Gamma_{k+1}\right\}
\end{array}\right] \leq 0,} \\
{\left[\begin{array}{cc}
-\tilde{\Theta}_{k} & \bar{\Pi}_{k}^{\mathrm{T}} \mathcal{L}_{n, i}^{\mathrm{T}} \\
\mathcal{L}_{n, i} \bar{\Pi}_{k} & -\Gamma_{k+1}
\end{array}\right] \leq 0}
\end{gathered}
$$

where

$$
\begin{aligned}
\bar{\vartheta}_{k} & =\left(I_{\epsilon} \otimes\left(\mathcal{L}_{n, i} \mathcal{D}_{k}\right)\right) \operatorname{col}_{\epsilon}\left\{\vartheta_{i, k}\right\}, \\
\bar{\pi}_{k} & =\left(I_{\varepsilon} \otimes\left(\mathcal{L}_{n, i} \mathcal{E}_{k}\right)\right) \operatorname{col}_{\varepsilon}\left\{\pi_{i, k}\right\}, \\
\tilde{\Theta}_{1} & =1-\left(\gamma_{i, k}+\lambda_{i, k}\right)-\sum_{i=1}^{N}\left(\tau_{i, k}^{(1)}+\tau_{i, k}^{(2)}\right),
\end{aligned}
$$

$$
\begin{aligned}
\tilde{\Theta}_{2} & =\sum_{i=1}^{N} \tau_{i, k}^{(1)} \mathcal{L}_{n, i}^{\mathrm{T}} \mathcal{L}_{n, i}, \\
\tilde{\Theta}_{3} & =\sum_{i=1}^{N} \tau_{i, k}^{(2)} \mathcal{L}_{q, i}^{\mathrm{T}} \Omega_{i, k}^{-1} \mathcal{L}_{q, i}, \\
\tilde{\Theta}_{k} & =\tau_{k}^{(3)} \Psi_{k}+\operatorname{diag}\left\{\tilde{\Theta}_{1}, \tilde{\Theta}_{2}, \tilde{\Theta}_{3}, 0\right\}
\end{aligned}
$$

with $F_{k} \in \mathbb{R}^{n \times n}$ being a factorization of $\Gamma_{k}$ (i.e., $\Gamma_{k}=$ $F_{k} F_{k}^{\mathrm{T}}$ ).

Proof: First of all, denote $\tilde{x}_{i, k} \triangleq x_{i, k}-\bar{x}_{k}$ and $\tilde{x}_{k} \triangleq$ $\operatorname{col}_{N}\left\{\tilde{x}_{i, k}\right\}$. By subtracting (14) from (1), we obtain

$$
\begin{aligned}
\tilde{x}_{i, k+1}= & x_{i, k+1}-\bar{x}_{k+1} \\
= & A_{k} x_{i, k}+B_{k}\left(K_{k}\left(\eta_{i, k}+e_{i, k}\right)\right)+D_{k} w_{i, k} \\
& -\left(A_{k} \bar{x}_{k}+\frac{1}{N}\left(\mathbf{1}_{N}^{\mathrm{T}} \otimes\left(B_{k} K_{k}\right)\right) e_{k}\right) .
\end{aligned}
$$

Taking (12) into consideration, we can easily acquire

$$
\begin{aligned}
\tilde{x}_{k+1}= & x_{k+1}-\left(\mathbf{1}_{N} \otimes I_{n}\right) \bar{x}_{k+1} \\
= & \left(I_{N} \otimes A_{k}+\mathcal{H}_{k} \otimes\left(B_{k} K_{k} G_{1} C_{k}\right)\right) x_{k} \\
& +\left(\mathcal{H}_{k} \otimes\left(B_{k} K_{k}\right)\right) \varphi_{k}+\left(I_{N} \otimes D_{k}\right) w_{k} \\
& +\left(\mathcal{H}_{k} \otimes\left(B_{k} K_{k} E_{k}\right)\right) v_{k}+\left(I_{N} \otimes\left(B_{k} K_{k}\right)\right) e_{k} \\
& -\left(\mathbf{1}_{N} \otimes I_{n}\right) A_{k} \bar{x}_{k}-\frac{1}{N}\left(\left(\mathbf{1}_{N} \mathbf{1}_{N}^{\mathrm{T}}\right) \otimes\left(B_{k} K_{k}\right)\right) e_{k} \\
= & \left(I_{N} \otimes A_{k}+\mathcal{H}_{k} \otimes\left(B_{k} K_{k} G_{1} C_{k}\right)\right) x_{k} \\
& +\mathcal{D}_{k} w_{k}+\mathcal{E}_{k} v_{k}+\left(\mathcal{H}_{k} \otimes\left(B_{k} K_{k}\right)\right) \varphi_{k} \\
& -\left(\mathbf{1}_{N} \otimes A_{k}\right) \bar{x}_{k}-\left(\mathcal{N}_{k} \otimes\left(B_{k} K_{k}\right)\right) e_{k} .
\end{aligned}
$$

The rest of the proof is performed by induction. It follows directly from (16) that, when $k=0, \mathfrak{D}_{i, 0} \leq \Gamma_{0}(\forall i \in \mathscr{V})$ is satisfied. Supposing that $\mathfrak{D}_{i, k} \leq \Gamma_{k}$ holds at time instant $k$, it remains to prove that $\mathfrak{D}_{i, k+1} \leq \Gamma_{k+1}$ also holds with the condition given in the theorem. Next, it can be easily verified that if

$$
\mathbb{E}\left\{\left(x_{i, k}-\bar{x}_{k}\right)\left(x_{i, k}-\bar{x}_{k}\right)^{\mathrm{T}}\right\} \leq \Gamma_{k},
$$

then there exists $z_{i, k} \in \mathbb{R}^{n}$ with $\mathbb{E}\left\{z_{i, k} z_{i, k}^{\mathrm{T}}\right\} \leq I_{n}$ such that

$$
x_{i, k}=\bar{x}_{k}+F_{k} z_{i, k}
$$

where $F_{k} \in \mathbb{R}^{n \times n}$ is a factorization of $\Gamma_{k}$ (i.e., $\Gamma_{k}=F_{k} F_{k}^{\mathrm{T}}$ ). Hence, with $z_{k} \triangleq \operatorname{col}_{N}\left\{z_{i, k}\right\}$, it follows from (27) that

$$
x_{k}=\left(\mathbf{1}_{N} \otimes I_{n}\right) \bar{x}_{k}+\left(I_{N} \otimes F_{k}\right) z_{k} .
$$

With the help of (28), we obtain from (25) that

$$
\begin{aligned}
\tilde{x}_{k+1}= & x_{k+1}-\left(\mathbf{1}_{N} \otimes I_{n}\right) \bar{x}_{k+1} \\
= & \left(I_{N} \otimes A_{k}+\mathcal{H}_{k} \otimes\left(B_{k} K_{k} G_{1} C_{k}\right)\right) \\
& \times\left(\left(\mathbf{1}_{N} \otimes I_{n}\right) \bar{x}_{k}+\left(I_{N} \otimes F_{k}\right) z_{k}\right) \\
& +\left(\mathcal{H}_{k} \otimes\left(B_{k} K_{k}\right)\right) \varphi_{k}+\left(I_{N} \otimes D_{k}\right) w_{k} \\
& +\left(\mathcal{H}_{k} \otimes\left(B_{k} K_{k} E_{k}\right)\right) v_{k}-\left(\mathbf{1}_{N} \otimes A_{k}\right) \bar{x}_{k} \\
& -\left(\mathcal{N}_{k} \otimes\left(B_{k} K_{k}\right)\right) e_{k} \\
= & \left(\mathbf{1}_{N} \otimes A_{k}\right) \bar{x}_{k}+\left(I_{N} \otimes\left(A_{k} F_{k}\right)\right) z_{k} \\
& +\left(\mathcal{H}_{k} \otimes\left(B_{k} K_{k} G_{1} C_{k} F_{k}\right)\right) z_{k}+\left(I_{N} \otimes D_{k}\right) w_{k} \\
& +\left(\mathcal{H}_{k} \otimes\left(B_{k} K_{k}\right)\right) \varphi_{k}+\left(\mathcal{H}_{k} \otimes\left(B_{k} K_{k} E_{k}\right)\right) v_{k} \\
& -\left(\mathbf{1}_{N} \otimes A_{k}\right) \bar{x}_{k}-\left(\mathcal{N}_{k} \otimes\left(B_{k} K_{k}\right)\right) e_{k} \\
= & \left(I_{N} \otimes D_{k}\right) w_{k}+\left(\mathcal{H}_{k} \otimes\left(B_{k} K_{k} E_{k}\right)\right) v_{k} \\
& +\left(I_{N} \otimes\left(A_{k} F_{k}\right)+\mathcal{H}_{k} \otimes\left(B_{k} K_{k} G_{1} C_{k} F_{k}\right)\right) z_{k}
\end{aligned}
$$




$$
\begin{aligned}
& -\left(\mathcal{N}_{k} \otimes\left(B_{k} K_{k}\right)\right) e_{k}+\left(\mathcal{H}_{k} \otimes\left(B_{k} K_{k}\right)\right) \varphi_{k} \\
= & \left(I_{N} \otimes\left(A_{k} F_{k}\right)+\mathcal{H}_{k} \otimes\left(B_{k} K_{k} G_{1} C_{k} F_{k}\right)\right) z_{k} \\
& -\left(\mathcal{N}_{k} \otimes\left(B_{k} K_{k}\right)\right) e_{k}+\left(\mathcal{H}_{k} \otimes\left(B_{k} K_{k}\right)\right) \varphi_{k} \\
& +\mathcal{D}_{k} w_{k}+\mathcal{E}_{k} v_{k} .
\end{aligned}
$$

Subsequently, by denoting

$$
\begin{aligned}
& \xi_{k} \triangleq\left[\begin{array}{llll}
1 & z_{k}^{\mathrm{T}} & e_{k}^{\mathrm{T}} & \varphi_{k}^{\mathrm{T}}
\end{array}\right]^{\mathrm{T}}, \\
& \Pi_{k} \triangleq\left[\mathcal{D}_{k} w_{k}+\mathcal{E}_{k} v_{k} \bar{\Pi}_{12}\right. \\
& \left.-\mathcal{N}_{k} \otimes\left(B_{k} K_{k}\right) \mathcal{H}_{k} \otimes\left(B_{k} K_{k}\right)\right],
\end{aligned}
$$

we can further express $\tilde{x}_{k+1}$ in (29) as follows:

$$
\tilde{x}_{k+1}=x_{k+1}-\left(\mathbf{1}_{N} \otimes I_{n}\right) \bar{x}_{k+1}=\Pi_{k} \xi_{k} .
$$

Next, it follows from Lemma 3 that

$$
\begin{aligned}
\mathbb{E}\left\{z_{i, k} z_{i, k}^{\mathrm{T}}\right\} \leq I_{n} & \Rightarrow \mathbb{E}\left\{z_{i, k}^{\mathrm{T}} z_{i, k}\right\} \leq 1 \\
& \Rightarrow \mathbb{E}\left\{\xi_{k}^{\mathrm{T}} \mathcal{T}_{1, k} \xi_{k}\right\} \leq 0
\end{aligned}
$$

where $\mathcal{T}_{1, k}$ is defined previously.

By the same token, we can know from (11) that the vector $e_{i, k}$ satisfies

$$
e_{i, k}^{\mathrm{T}} \Omega_{i, k}^{-1} e_{i, k} \leq 1
$$

which can be described by $\xi_{k}$ as follows:

$$
\xi_{k}^{\mathrm{T}} \mathcal{T}_{2, k} \xi_{k} \leq 0
$$

where $\mathcal{T}_{2, k}$ is defined previously.

Similarly, inequality (6) which characterizes the constraints resulting from the sensor saturations can be rewritten as

$$
\left(\left(I_{N} \otimes I_{n}\right) \varphi_{k}\right)^{\mathrm{T}}\left(\varphi_{k}-\left(I_{N} \otimes\left(G C_{k}\right)\right) x_{k}\right) \leq 0 .
$$

Substituting (27) into (36) leads to

$$
\begin{aligned}
\varphi_{k}^{\mathrm{T}}\left(I_{N} \otimes I_{n}\right) & \left(\varphi_{k}-\left(I_{N} \otimes\left(G C_{k}\right)\right)\right. \\
& \left.\times\left(\left(\mathbf{1}_{N} \otimes I_{n}\right) \bar{x}_{k}+\left(I_{N} \otimes F_{k}\right) z_{k}\right)\right) \leq 0,
\end{aligned}
$$

which can be equivalently expressed by $\xi_{k}$ as

$$
\xi_{k}^{\mathrm{T}} \Psi_{k} \xi_{k} \leq 0
$$

with $\Psi_{k}$ being defined previously.

So far, in terms of the vector $\xi_{k}$, we have converted all the constraints imposed on the time-varying MAS (1)-(2) into certain inequalities (i.e. (33), (35) and (37)). It now remains to show that $\Gamma_{k+1} \leq 1$ holds if the condition of this theorem is satisfied at time instant $k$. To this end, by means of Lemma 3 , the set of RLMIs (22) is feasible if and only if

$$
-\tilde{\Theta}_{k}+\bar{\Pi}_{k}^{\mathrm{T}} \mathcal{L}_{n, i}^{\mathrm{T}} \Gamma_{k+1}^{-1} \mathcal{L}_{n, i} \bar{\Pi}_{k} \leq 0 .
$$

For brevity of later development, we denote

$$
\vec{\Pi}_{k} \triangleq \bar{\Pi}_{k}^{\mathrm{T}} \mathcal{L}_{n, i}^{\mathrm{T}} \Gamma_{k+1}^{-1} \mathcal{L}_{n, i} \bar{\Pi}_{k} .
$$

Substituting (23) into (38) yields

$$
\xi_{k}^{\mathrm{T}}\left(\vec{\Pi}_{k}-\tau_{k}^{(3)} \Psi_{k}-\operatorname{diag}\left\{\tilde{\Theta}_{1}, \tilde{\Theta}_{2}, \tilde{\Theta}_{3}, 0\right\}\right) \xi_{k} \leq 0 .
$$

After some tedious but straightforward manipulations, we arrive at

$$
\xi_{k}^{\mathrm{T}}\left(\vec{\Pi}_{k}+\breve{\Pi}_{k}-\tau_{k}^{(3)} \Psi_{k}-\sum_{i=1}^{N}\left(\tau_{i, k}^{(1)} \mathcal{T}_{1, k}+\tau_{i, k}^{(2)} \mathcal{T}_{2, k}\right)\right) \xi_{k} \leq 0
$$

where $\breve{\Pi}_{k} \triangleq \operatorname{diag}\left\{\gamma_{i, k}+\lambda_{i, k}, 0,0,0\right\}-\operatorname{diag}\{1,0,0,0\}$.

By Lemma 2, it follows from (33), (35), (37) and (39) that

$$
\xi_{k}^{\mathrm{T}}\left(\vec{\Pi}_{k}+\breve{\Pi}_{k}\right) \xi_{k} \leq 0 .
$$

According to Lemma 3, the set of RLMIs (20) holds if and only if

$$
-\gamma_{i, k}+\sum_{l=1}^{\epsilon} \vartheta_{l, k}^{\mathrm{T}} \mathcal{D}_{k}^{\mathrm{T}} \mathcal{L}_{n, i}^{\mathrm{T}} \Gamma_{k+1}^{-1} \mathcal{L}_{n, i} \mathcal{D}_{k} \vartheta_{l, k} \leq 0
$$

which, by properties of matrix trace, are equivalent to

$$
-\gamma_{i, k}+\sum_{l=1}^{\epsilon} \operatorname{tr}\left[\mathcal{D}_{k}^{\mathrm{T}} \mathcal{L}_{n, i}^{\mathrm{T}} \Gamma_{k+1}^{-1} \mathcal{L}_{n, i} \mathcal{D}_{k} \vartheta_{l, k} \vartheta_{l, k}^{\mathrm{T}}\right] \leq 0 .
$$

Since $\mathcal{W}_{k}=\sum_{l=1}^{\epsilon} \vartheta_{l, k} \vartheta_{l, k}^{\mathrm{T}}$, inequalities (42) imply

$$
\operatorname{tr}\left[\mathscr{D}_{k}\right]=\operatorname{tr}\left[\mathcal{D}_{k}^{\mathrm{T}} \mathcal{L}_{n, i}^{\mathrm{T}} \Gamma_{k+1}^{-1} \mathcal{L}_{n, i} \mathcal{D}_{k} \mathcal{W}_{k}\right] \leq \gamma_{i, k}
$$

Along the similar line, it can be derived from RLMIs (21) that

$$
\operatorname{tr}\left[\mathscr{E}_{k}\right]=\operatorname{tr}\left[\mathcal{E}_{k}^{\mathrm{T}} \mathcal{L}_{n, i}^{\mathrm{T}} \Gamma_{k+1}^{-1} \mathcal{L}_{n, i} \mathcal{E}_{k} \mathcal{V}_{k}\right] \leq \lambda_{i, k}
$$

Denoting $\tilde{\Pi}_{k} \triangleq\left[\mathcal{D}_{k} w_{k}+\mathcal{E}_{k} v_{k} \quad \begin{array}{lll}0 & 0\end{array}\right]$ and taking into account the statistical properties of random variables $w_{k}$ and $v_{k}$, we obtain

$$
\begin{aligned}
& \mathbb{E}\left\{\xi_{k}^{\mathrm{T}} \Pi_{k}^{\mathrm{T}} \mathcal{L}_{n, i}^{\mathrm{T}} \Gamma_{k+1}^{-1} \mathcal{L}_{n, i} \Pi_{k} \xi_{k}\right\} \\
= & \xi_{k}^{\mathrm{T}} \vec{\Pi}_{k} \xi_{k}+\mathbb{E}\left\{\xi_{k}^{\mathrm{T}} \tilde{\Pi}_{k}^{\mathrm{T}} \mathcal{L}_{n, i}^{\mathrm{T}} \Gamma_{k+1}^{-1} \mathcal{L}_{n, i} \tilde{\Pi}_{k} \xi_{k}\right\} \\
= & \xi_{k}^{\mathrm{T}} \vec{\Pi}_{k} \xi_{k}+\xi_{k}^{\mathrm{T}} \operatorname{diag}\left\{\operatorname{tr}\left[\mathscr{D}_{k}\right]+\operatorname{tr}\left[\mathscr{E}_{k}\right], 0,0,0\right\} \xi_{k} .
\end{aligned}
$$

Therefore, it can be verified from inequalities (43), (44) and (40) that the following is true:

$$
\mathbb{E}\left\{\xi_{k}^{\mathrm{T}} \Pi_{k}^{\mathrm{T}} \mathcal{L}_{n, i}^{\mathrm{T}} \Gamma_{k+1}^{-1} \mathcal{L}_{n, i} \Pi_{k} \xi_{k}\right\}-1 \leq 0 .
$$

Applying now Lemma 3 to inequalities (46), we acquire

$$
\mathbb{E}\left\{\mathcal{L}_{n, i} \Pi_{k} \xi_{k} \xi_{k}^{\mathrm{T}} \Pi_{k}^{\mathrm{T}} \mathcal{L}_{n, i}^{\mathrm{T}}\right\} \leq \Gamma_{k+1}
$$

which implies that $\mathfrak{D}_{i, k+1} \leq \Gamma_{k+1}(i=1, \ldots, N)$ also hold and the induction is now accomplished. Consequently, MAS (1)-(2) reaches mean-square consensus and the proof is thus complete.

It follows from Theorem 1 that the desired control protocols could be a set if non-empty. An interesting issue would be to look for certain optimal protocol among the feasible set based on some criteria of engineering significance. In the following, two optimization problems are discussed in order to seek the locally best consensus performance and locally lowest triggering frequency, respectively. 


\section{B. Optimization Problems}

Problem 1: Minimization of $\left\{\Gamma_{k}\right\}_{k>0}$ (in the sense of matrix trace) subject to fixed couple $\left(\mathscr{G},\left\{\Omega_{i, k}\right\}\right)$ for locally best consensus performance

Corollary 1: Let the pair $\left(\mathscr{G},\left\{\Omega_{i, k}\right\}\right)$ be given. A sequence of minimized $\left\{\Gamma_{k}\right\}_{k>0}$ (in the sense of matrix trace) can be obtained if there exist real-valued matrices $\left\{K_{k}\right\}_{k \geq 0}$, positive scalars $\left\{\gamma_{i, k}\right\}_{k \geq 0}$ and $\left\{\lambda_{i, k}\right\}_{k \geq 0}$, non-negative scalars $\left\{\tau_{i, k}^{(1)}\right\}_{k \geq 0},\left\{\tau_{i, k}^{(2)}\right\}_{k \geq 0}$ and $\left\{\tau_{k}^{(3)}\right\}_{k \geq 0}(i=1,2, \ldots N)$ solving the following optimization problem:

$$
\begin{gathered}
\min _{\Gamma_{k+1}, K_{k}, \gamma_{i, k}, \lambda_{i, k}, \tau_{i, k}^{(1)}, \tau_{i, k}^{(2)}, \tau_{k}^{(3)}} \operatorname{tr}\left[\Gamma_{k+1}\right] \\
\text { s.t. (20) - (22). }
\end{gathered}
$$

Problem 2: Maximization of $\left\{\Omega_{i, k}\right\}_{k \geq 0}$ (in the sense of matrix trace) subject to fixed couple $\left(\mathscr{G},\left\{\Gamma_{k}\right\}\right)$ for locally lowest triggering frequency

Corollary 2: Let the pair $\left(\mathscr{G}, \Gamma_{k}\right)$ be given. A sequence of maximized $\Omega_{i, k}$ (in the sense of matrix trace) is guaranteed if there exist real-valued matrices $\left\{K_{k}\right\}_{k \geq 0}$ and $\left\{\Upsilon_{i, k}\right\}_{k \geq 0}$, positive scalars $\left\{\gamma_{i, k}\right\}_{k>0}$ and $\left\{\lambda_{i, k}\right\}_{k>0}$, non-negative scalars $\left\{\tau_{i, k}^{(1)}\right\}_{k \geq 0},\left\{\tau_{i, k}^{(2)}\right\}_{k \geq 0}$ and $\left\{\tau_{k}^{(3)}\right\}_{k \geq 0}(i=1,2, \ldots, N)$ solving the following optimization problem:

$$
\begin{gathered}
\min _{K_{k}, \Upsilon_{i, k}, \gamma_{i, k}, \lambda_{i, k}, \tau_{i, k}^{(1)}, \tau_{i, k}^{(2)}, \tau_{k}^{(3)}} \operatorname{tr}\left[\sum_{i=1}^{N} \alpha_{i} \Upsilon_{i, k}\right] \\
\text { s.t. (20) - (21) \& }\left[\begin{array}{cc}
-\hat{\Theta}_{k} & * \\
\mathcal{L}_{n, i} \bar{\Pi}_{k} & -\Gamma_{k+1}
\end{array}\right] \leq 0
\end{gathered}
$$

where

$$
\hat{\Theta}_{k}=\operatorname{diag}\left\{\tilde{\Theta}_{1}, \tilde{\Theta}_{2}, \sum_{i=1}^{N} \mathcal{L}_{q, i}^{\mathrm{T}} \tau_{i, k}^{(2)} \Upsilon_{i, k} \mathcal{L}_{q, i}, 0\right\}+\tau_{k}^{(3)} \Psi_{k}
$$

and $\alpha_{i}>0$ are weighting scalars satisfying $\sum_{i=1}^{N} \alpha_{i}=1$. The triggering threshold matrix $\Omega_{i, k}$ can be computed by $\Omega_{i, k}=$ $\Upsilon_{i, k}^{-1}$.

The proofs of Corollaries 1-2 are straightforward and thus omitted.

Remark 2: Based on Theorem 1, Corollaries 1-2 convert the original optimization problems to certain eigenvalues problems (EVPs) for minimizing the sum of the eigenvalues (which is equivalent to the matrix trace) of certain variables subject to the RLMIs constraints. As discussed in [3], such EVPs can be solved numerically using the interior-point method efficiently. Moreover, note that the control law is linear function of the output deviations. Therefore, the suboptimal control algorithms developed in Corollaries 1-2 can only be applied to the linear output feedback control case. The corresponding optimal consensus control problem via nonlinear feedback is one of problems deserving our further investigation.

Remark 3: So far, we have shown that 1) the solvability of the addressed mean-square consensus problem is cast into the feasibility of a set of RLMIs; and 2) the proposed strategy is capable of making trade-off between the consensus accuracy (characterized by $\Gamma_{k}$ ) and the triggering frequency (illustrated by $\Omega_{i, k}$ ) by making full use of the design flexibility. Moreover, our main results can also be extended to the filtering/control problems for systems discussed in [16], [25]. In particular, it is worth pointing out that another one of our possible research topics in future is to investigate MASs with much more complicated dynamics such as the heterogeneous structures in [12], the Markovian jump parameters in [15] and the randomevent triggering mechanism in [10] due to their engineering significance.

Remark 4: It should be pointed out that, within the proposed framework, the feedback gain $K_{k}$ needs to be calculated first by using the global information on the topology $\mathcal{H}_{k}$ before the implementation. Then, according to the obtained control protocol, the agents will reach the desired consensus by using the neighbors' information only. In this consensus process, the global information is no longer required and, therefore, the multi-agent system works in a distributed way. It is worth mentioning that there have been some research papers coping with the MAS control problems by utilizing the global information (e. g., the topology structure or the maximum/minimum eigenvalues of Laplacian matrices), see [24] for example. [4] is another quintessential example where both centralized and distributed approaches have been developed to deal with the event-triggered control for multi-agent systems. Nevertheless, it would be interesting to develop a framework within which the control protocol can be designed only using the neighbors' information of the agents, and this will be one of our future research topics.

\section{ILLUSTRATIVE EXAMPLE}

Consider a multi-agent systems with following parameters:

$$
\begin{aligned}
A_{k} & =\left[\begin{array}{cc}
1+0.2 \sin (0.3 k) & 0.02+0.02 \sin (k) \\
0.02 & 1+0.2 \sin (2 k)
\end{array}\right], \\
B_{k} & =\left[\begin{array}{cc}
0.3+0.15 \cos (3 k) \\
0.3+0.12 e^{-k}
\end{array}\right], D_{k}=\left[\begin{array}{c}
0.3+0.06 \cos (3 k) \\
0.03
\end{array}\right], \\
C_{k} & =\left[\begin{array}{cc}
0.2+0.03 \sin (k) & 0.25+0.01 \cos (4 k)
\end{array}\right], \\
E_{k} & =0.3+0.03 \sin (k), W_{i, k}=V_{i, k}=1, G_{1}=0.9, G_{2}=1 .
\end{aligned}
$$

Let there be 4 agents connected according to an undirected graph $\mathscr{G}$ with the associated matrix $\mathcal{H}$ set by

$$
\mathcal{H}=\left[\begin{array}{cccc}
-4 & 1 & 2 & 1 \\
1 & -4 & 1 & 2 \\
2 & 1 & -4 & 1 \\
1 & 2 & 1 & -4
\end{array}\right]
$$

In this simulation, the saturation threshold value $r_{\max }=$ 5. The function $\varphi\left(C_{k} x_{i, k}\right)$ in (5) can then be described by $\varphi\left(C_{k} x_{i, k}\right)=\kappa\left(C_{k} x_{i, k}\right)-0.9 C_{k} x_{i, k}$.

Set the initial values of agents' states and the initial $\Gamma_{0}$ as follows:

$$
\begin{aligned}
& x_{1,0}=\left[\begin{array}{c}
20 \\
5
\end{array}\right], x_{2,0}=\left[\begin{array}{c}
25 \\
15
\end{array}\right], x_{3,0}=\left[\begin{array}{l}
10 \\
20
\end{array}\right], \\
& x_{4,0}=\left[\begin{array}{c}
5 \\
30
\end{array}\right], \Gamma_{0}=\left[\begin{array}{cc}
147 & -75 \\
-75 & 226
\end{array}\right] .
\end{aligned}
$$

Then, it can be easily checked that the initial condition (16) is satisfied. By implementing the schemes proposed in 
Corollaries 1-2, the simulation results are shown in Table I, Table II and Figs. 1-6.

Table I presents some of the output feedback controller gains by solving the RLMIs in Corollaries 1-2. It can be seen from Figs. 1-4 that the trajectories of each agent in Problem 1 are much closer to the average state than those in Problem 2, which indicates that the algorithm proposed in Corollary 1 leads to a better consensus performance. As far as the triggering frequency is concerned, the total triggering times are shown, with a comparison to the conventional time-based strategy, in Table II for both optimization problems. It can be observed that i) the proposed event-triggering mechanism can effectively reduce the triggering frequency; and ii) the total triggering times in Problem 2 are less than those in Problem 1 which implies, as we anticipate, that the triggering frequency can be further reduced if we implement the strategy provided in Corollary 2. Such a finding can be further verified via the comparison between Fig. 5 and Fig. 6.

TABLE I

THE OUTPUT-FEEDBACK CONTROLLER GAINS AT EACH TIME STEP

\begin{tabular}{ccccccc}
\hline Time & $k=0$ & $k=1$ & $k=2$ & $k=3$ & $k=4$ & $\cdots$ \\
\hline$K_{k}(O P 1)$ & 0.35 & 0.28 & 0.16 & 0.25 & 0.36 & $\cdots$ \\
\hline$K_{k}(O P 2)$ & 0.27 & 0.46 & 0.35 & 0.57 & 0.22 & $\cdots$ \\
\hline
\end{tabular}

TABLE II

COMPARISON OF TRIGGERING TIMES

\begin{tabular}{cccc}
\hline Methodology & Time-based method & Problem 1 & Problem 2 \\
\hline Triggering times & 180 & 150 & 112 \\
\hline
\end{tabular}

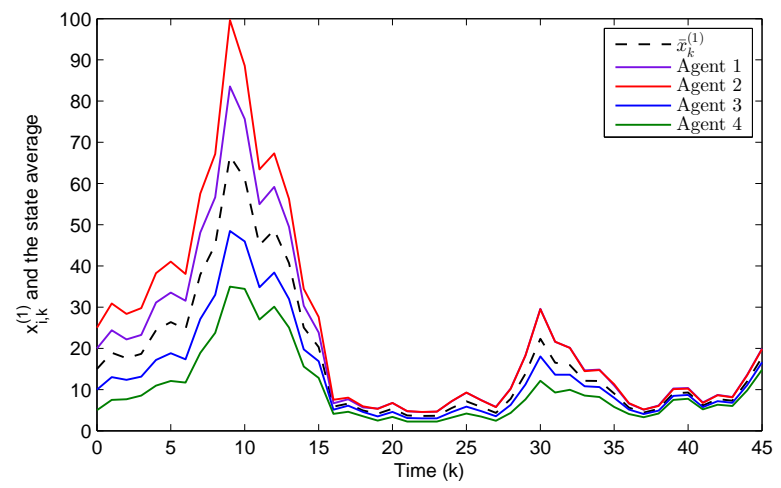

Fig. 1. The trajectories of $x_{i, k}^{(1)}$ and the average $\bar{x}_{k}^{(1)}$ for Problem 1 .

\section{CONCLUSION}

In this paper, the event-triggered mean-square consensus control problem has been investigated for a class of discrete time-varying stochastic multi-agent system subject to sensor saturations. First, a new definition of mean-square consensus has been presented for the addressed MAS to characterize the transient consensus behavior. Then, by means of an RLMI approach, sufficient conditions have been established for the existence of the desired controller. Within the established framework, two optimization problems have been discussed to optimize the consensus performance and triggering frequency, respectively. Finally, an illustrative example has been exploited to show the effectiveness of the proposed control scheme.

\section{REFERENCES}

[1] M. Basin, S. Elvira-Ceja, and E. Sanchez, Central suboptimal meansquare $H_{\infty}$ controller design for linear stochastic time-varying systems, Int. J. Syst. Science, vol. 42, no. 5, pp. 821-827, 2011.

[2] J. G. Bender, An overview of systems studies of automated highway systems, IEEE Trans. Veh. Technol., vol. 40, no. 1, pp. 82-99, 1991.

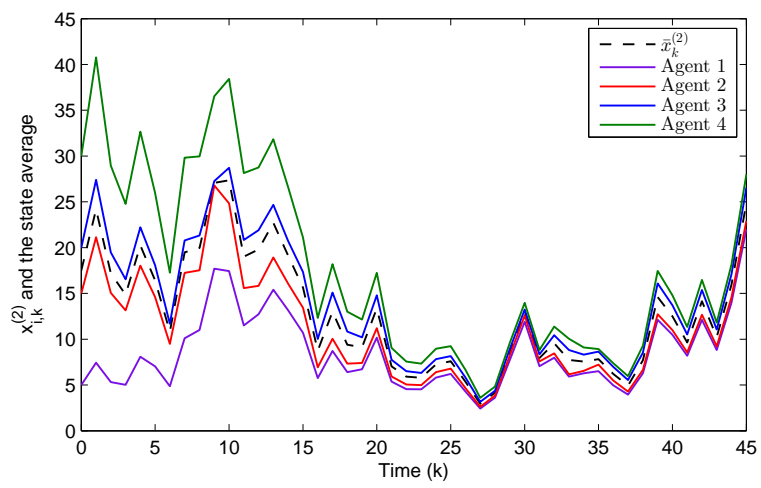

Fig. 2. The trajectories of $x_{i, k}^{(2)}$ and the average $\bar{x}_{k}^{(2)}$ for Problem 1.

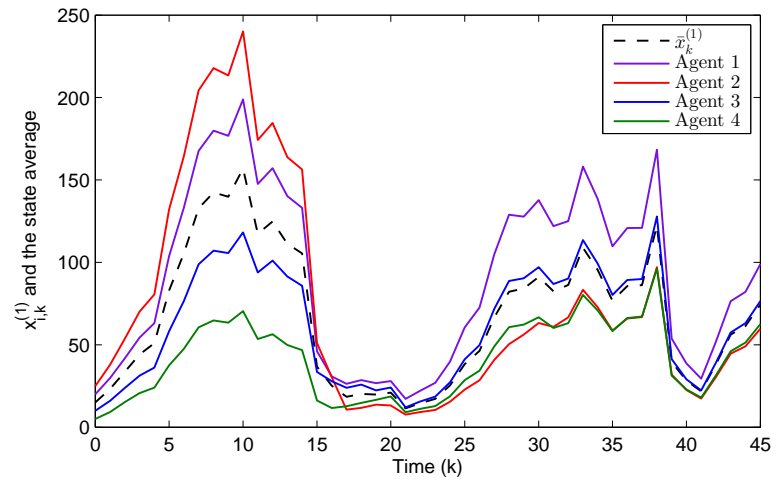

Fig. 3. The trajectories of $x_{i, k}^{(1)}$ and the average $\bar{x}_{k}^{(1)}$ for Problem 2.

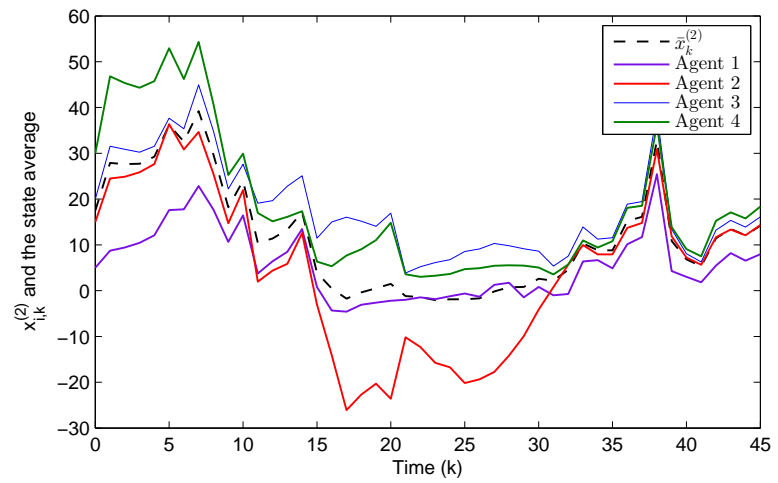

Fig. 4. The trajectories of $x_{i, k}^{(2)}$ and the average $\bar{x}_{k}^{(2)}$ for Problem 2. 
[3] S. Boyd, L. Ghaoui, E. Feron and V. Balakrishnan, Linear matrix inequalities in system and control theory, Philadelphia: SIAM Studies in Applied Mathematics, 1994.

[4] D. V. Dimarogonas, E. Frazzoli and K. H. Johansson, Distributed eventtriggered control for multi-agent systems, IEEE Trans. Autom. Control, vol. 57, no. 5, pp. 1291-1297, 2012.

[5] D. Ding, Z. Wang, B. Shen and G. Wei, Event-triggered consensus control for discrete-time stochastic multi-agent systems: the input-tostate stability in probability, Automatica, vol. 62, pp. 284-291, 2015.

[6] H. Dong, Z. Wang, B. Shen and D. Ding, Variance-constrained $H_{\infty}$ control for a class of nonlinear stochastic discrete time-varying systems: the event-triggered design, Automatica, vol. 72, pp. 28-36, 2016.

[7] Y. Fan, G. Feng, Y. Wang and C. Song, Distributed event-triggered control of multi-agent systems with combinational measurements, $\mathrm{Au}$ tomatica, vol. 49, no. 2, pp. 671-675, 2013.

[8] E. Gershon, U. Shaked and I. Yaesh, $H_{\infty}$ control and estimation of state-multiplicative linear systems, Springer-Verlage London, 2005.

[9] G. Guo, L. Ding and Q.-L. Han, A distributed event-triggered transmission strategy for sampled-data consensus of multi-agent systems, Automatica, vol. 50, pp. 1489-1496, 2014.

[10] G. Guo, Z. Lu and Q.-L. Han, Control with Markov sensors/actuators assignment, IEEE Trans. Autom. Control, vol. 57, no. 7, pp. 1799-1804, 2012.

[11] J. Kim, J. Yang, J. Kim and H. Shim, Practical consensus for heterogeneous linear time-varying multi-agent systems, Proc. 12th Int. Conf. Control, Automation, Syst., Korea, pp. 23-28, 2012.

[12] H. Kim, H. Shim and J. H. Seo, Output consensus of heterogeneous uncertain linear multi-agent systems, IEEE Trans. Autom. Control, vol. 56, no. 1, pp. 200-206, 2011.

[13] H. Kopetz, Event-triggered versus time-triggered real-time systems, Operating Systems of the 90 s and Beyond, Lecture Notes in Computer Science, vol. 563, pp. 86-101, 1991.

[14] Z. Li, Z. Duan and F. L. Lewis, Distributed robust consensus control

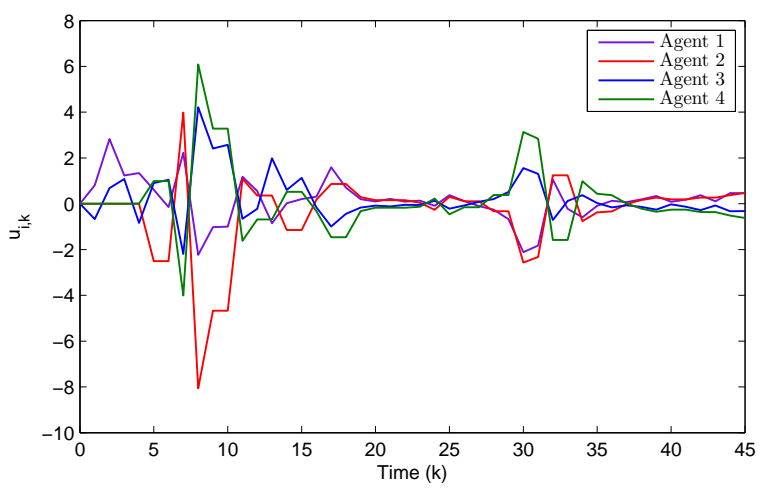

Fig. 5. The control input $u_{i, k}$ for Problem 1 .

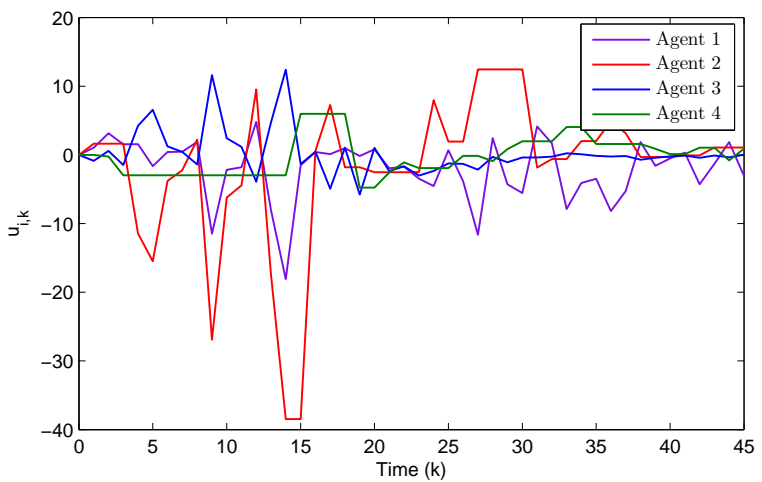

Fig. 6. The control input $u_{i, k}$ for Problem 2. of multi-agent systems with heterogeneous matching uncertainties, $\mathrm{Au}$ tomatica, vol. 50, no. 3, pp. 883-889, 2014.

[15] Y. Liu, W. Liu, M. A. Obaid and I. A. Abbas, Exponential stability of Markovian jumping Cohen-Grossberg neural networks with mixed mode-dependent time-delays, Neurocomputing, vol. 177, pp. 409-415, 2016.

[16] D. Liu, Y. Liu and F. E. Alsaadi, A new framework for output feedback controller design for a class of discrete-time stochastic nonlinear system with quantization and missing measurement, Int. J. General Systems, vol. 45, no. 5, pp. 517-531, 2016.

[17] L. Ma, Z. Wang, H. K. Lam and N. Kyriakoulis, Distributed eventbased set-membership filtering for a class of nonlinear systems with sensor saturations over sensor networks, IEEE Trans. Cybernetics, DOI:10.1109/TCYB.2016.2582081, 2016.

[18] L. Ma, Z. Wang and H. K. Lam, Mean-square $H_{\infty}$ consensus Ccontrol for a class of nonlinear time-varying stochastic multiagent systems: the finite-horizon case, IEEE Trans. Systems, Man, and Cybernetics: Systems, DOI:10.1109/TSMC.2016.2531657, 2016.

[19] K. Sakurama, S. Azuma and T. Sugie, Distributed controllers for multiagent coordination via gradient-flow approach, IEEE Trans. Autom. Control, vol. 60, no. 6, pp. 1471-1485, 2015.

[20] G. S. Seyboth, D. V. Dimarogonas and K. H. Johansson, Event-based broadcasting for multi-agent average consensus, Automatica, vol. 49, no. 1, pp. 245-252, 2013.

[21] T. R. Smith, H. Hanssmann and N. E. Leonard, Orientation control of multiple underwater vehicles, In: Proc. 40th IEEE Conf. Decision and Control, pp. 4598-4603, 2001.

[22] J. D. Wolfe, D. F. Chichka and J. L. Speyer, Decentralized controllers for unmanned aerial vehicle formation flight, in Proc. AIAA Conf. Guidance, Navigation, and Control, 1996, AIAA Paper 96-3833.

[23] H. Yamaguchi, A cooperative hunting behavior by mobile robot troops, Int. J. Robotics Research, vol. 18, no. 9, pp. 931-940, 1999.

[24] K. You and L. Xie, Coordination of discrete-time multi-agent systems via relative output feedback, International Journal of Robust and Nonlinear Control, vol. 21, no. 13, pp. 1587-1605, 2011.

[25] J. Zhang, L. Ma and Y. Liu, Passivity analysis for discrete-time neural networks with mixed time-delays and randomly occurring quantization effects, Neurocomputing, DOI: 10.1016/j.neucom.2016.08.020, 2016.

[26] X. Zhang, L. Lu and G. Feng, Leader-follower consensus of time-varying nonlinear multi-agent systems, Automatica, vol. 52, pp. 8-14, 2015. 